\title{
Table of legislation and analogous instruments
}

\section{EUROPEAN UNION}

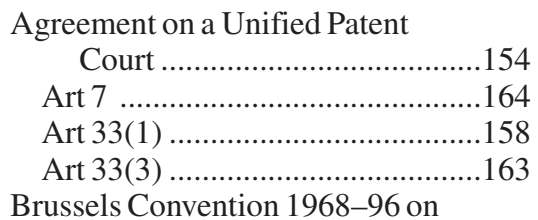
Jurisdiction and the Enforcement of Judgments in Civil and Commercial Matters viii, 27 , $29,31,75,77,123-4,268$, 347,349

Art 5(1) .75

Art 17 $31-2,75$

Art 17(2) . .35

Art 21 . .75

Art 25 .123

Brussels I Regulation, see Regulation 44/2001/EC; Regulation 1215/ 2012/EU

Charter of Fundamental Rights of the European Union 135,336 Art 7 316

Art 11 316

Art 47 135,137

Decision 2003/93/EC ...ix

Decision 2007/712/EC viii

Decision 2008/431/EC ...ix

Decision 2009/430/EC .viii

Decision 2009/941/EC ...ix

Decision 2011/432/EU ...ix

Directive 73/239/EEC relating to the taking-up and pursuit of the business of direct insurance other than life assurance .251
Directive 85/374/EEC concerning liability for defective products 310-11

Directive 86/653/EEC on commercial agents ........249, 254, 266, 276-7, 280-81

Art 17 $.276,280$ Art 18 276,280

Art 19 280

Directive 87/201/EEC on consumer credit 275

Directive 93/13/EEC on unfair terms in consumer contracts 270

Art 6 .254

Art 6(2) 261

Directive 93/7/EC on the return of cultural objects, Art 12 .254

Directive 95/46/EC on the protection of individuals with regard to the processing of personal data and on the free movement of such data .324

Directive $96 / 71 / \mathrm{EC}$ on the posting of workers

Recital 11 278

Art 3 254,278

Directive 96/9/EC on the legal protection of databases .............15

Directive 97/7/EC on distance contracts, Art 12 .254

Directive 98/26/EC on settlement finality

Art 1(a)

Art 2(a) 187

Directive $98 / 71 /$ EC on the legal protection of designs 153 
Directive 1999/44/EC on consumer goods, Art 7 .254

Directive 2000/31/EC on electronic commerce .......2, 4, 10-12, 20-21, Recital 19 $249,257-8,261-2,280,331$

Recital 23 $11,20,332$

Recital 90 .258

Art 1(4) $10,20,257,332$

Art 2(c)

Art 3 11,21

Art 3(1) $10,20,259$

Art 3(2)

Art 3(3) $10,20,257,259$

Art 3(4) 260

Art 4(1) 259

Art 17 .258

Directive 2001/24/EC on the reorganisation and winding up of credit institutions $187-8$

Preamble .188

Recital 24 .188

Art 24 187

Art 25 $187-8$

Art 27 $187-8$

Directive 2001/29/EC on copyright in the information society .... .. 15

Art 4 .15

Art 5 .15

Directive 2002/65/EC on the distance marketing of financial services, Art 12 .254

Directive 2004/39/EC on markets in financial instruments ...170, 185-6

Ann I 185

Directive 2006/123/EC on services in the internal market .....11, 257, 261

Recital 90 .21

Art 3(2) $11,21,257-8$

Art 16 . .11

Art 17(15) . .11

Art 17(5) .21

Directive 2008/122/EC on time share .269 Art 12 $254,261,270$

Directive 2008/48/EC on credit agreements, Art 22 .254
Directive 2008/95/EC to approximate the laws of the Member States relating to trade marks .153

Directive 2009/44/EC on settlement finality .187

Directive 2011/83/EU on consumer rights 249, 251, 256, 268-9,

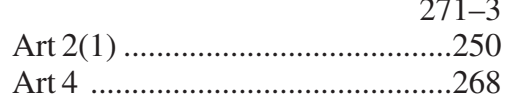

Luxembourg Protocol of 1971 on the interpretation of the Brussels Convention 1968

Regulation 2100/94/EC on Community plant variety rights ............16, 153

Arts 101-102 ….............................16

Art 101(2) .......................................17

Art 101(2)(c) ..................................17

Art 101(3) .....................................16

Regulation 1346/2000/EC on insolvency proceedings ......ix, 289

Regulation 44/2001/EC (Brussels I original version) ......viii, 1, 23, 85, $116,136,155,289,297,346$

Recital 1 ...................................128

Recital 3 ................................128

Recital 16 ..................................128

Recital 17 ...................................128

Art 2 .......................................346-54

Art 2(1) .........................................348

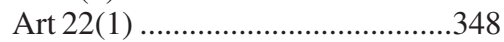

Art 22(2) ...................................361-4

Art 23 ..............25-6, 31, 35-7, 348

Art 27 .....................................39-40

Regulation 1206/2001/EC on

co-operation between the courts of the member states in the taking of evidence in civil or commercial matters

Regulation 6/2002/EC on Community designs $.16,153$

Arts $79-83$......................................16

Art 82(1)-(2) ..................................17

Art 82(3) .......................................17

Art 82(5) .......................................16

Art 83(1) ........................................ 17

Art 83(2) ........................................ 16 
Regulation 2201/2003/EC concerning jurisdiction and the recognition and enforcement of judgments in matrimonial matters and the matters of parental responsibility ix, 290, 292

Art 8(1) ix, 290-1

Regulation 805/2004/EC creating a European Enforcement Order for uncontested claims ..viii

Regulation 1896/2006/EC creating a European order for payment procedure viii

Regulation 861/2007/EC establishing a European Small Claims

Procedure ..viii

Regulation 864/2007/EC on the law applicable to non-contractual obligations (Rome II) ...ix, xi, 1-2, 18, 21, 190, 257-60, 283, 285-343, 355

Recital 6 .287

Recital 7 .296

Recital 13 .287

Recital 14 .287

Recital 16 .287

Recital 17 .297

Recital 18 .299

Recital 33 312-13

Recital 35 .20

Art 1(2) .285

$\operatorname{Art~1(2)(g)~}$

Art 1(3) $19,21,323$

Art 4 285-6, 311-13

Art 4(1) 18, 285-305, 311
Art 4(2) $301-3,313,355$ 286, 288, 292-4, 299-300, 303 286, 290, 292-3, 296-9,

Art 4(3) ....286, 288, 292-6, 298-304 Art 5 $18,285,304-11$

Art 5(1) 304-5

Art 5(1)(a) 305, 308-9

$\operatorname{Art~5(1)(b)~}$ $305,307,310$

$\operatorname{Art~5(1)(c)~}$ 305,308

Art 5(2) 304-5, 310

Arts 6-9 285

Art 6(1) 19,303

Art 6(2) ..303
Art 6(3) 19

Art 7 ..18

Art 8 19,313

Art 9 18

Art 10(3) .22

Art 11(3) 22

Art 12 299

Art 12(1) 299

Art 12(2) 299

Art 13 .19

Art 14 341

Art 15 311

Art 15(c) 311-13

Art 17 286

Art 21 311

Art 22 311

Art 23 288,306

Art 23(1) 288, 290, 306

Art 23(2) .290

Art 27 .20

Art 28 287

Art 30 324

Art 30(1) 324

Art 30(2) 324

Regulation 1393/2007/EC on the service in the Member States of judicial and extrajudicial documents in civil or commercial matters $\mathrm{X}$

Regulation 593/2008/EC on the law applicable to contractual obligations (Rome I)

Recital 6 253,262

Recital 11 $214,242,264$

Recital 12 215

Recital 13 ......222, 227, 229-32, 242

Recital 14 242

Recital 16 $172,191,216$

Recital 17 $183-4$

Recital 18 185,187

Recital 19 177

Recital 20 189

Recital 21 194

Recital 28

Recital 29 188

Recital 30 188

Recital 34 188

Recital 39 277 216 


\begin{tabular}{|c|c|}
\hline Recital 40 ..................10, 258, 261-3 & $, 269,272-9$, \\
\hline 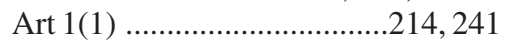 & $281-2$ \\
\hline 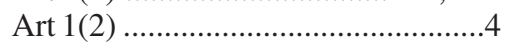 & $\operatorname{Art} 9(1) \ldots \ldots$ \\
\hline Art 1(2)(e) ....................... & Regulation 4/2009/EC on jurisdiction, \\
\hline 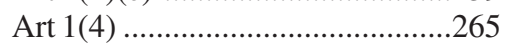 & applicable law, recognition and \\
\hline $.241,269$ & enforcement of decisions and \\
\hline ......6, 171, 218, 254, 262-7, & co-operation in matters relating to \\
\hline 269,273 & maintenance obligations .............ix \\
\hline Art $3(1) \ldots \ldots .$. & Regulation $207 / 2009 / \mathrm{EC}$ on the \\
\hline Art 3(2) ........215, 218, 226, 228, 234 & Community trade mark ....16, 153, \\
\hline Art 3(3) ..........215, 241, 265-7, 269, & \\
\hline 275,280 & $.163,167$ \\
\hline Art 3(4) .......263, 265, 267, 269-70, & Arts 94-98 \\
\hline $273,275,280,282$ & Art $95 \ldots . . .$. \\
\hline Art 4(1) ........171-3, 177-8, 180-89, & Art 96 . \\
\hline $192,195-6,215-16$ & Art 97 .. \\
\hline$\ldots .180,182,185,215$, & Art 97(1)-(2) . \\
\hline 217,271 & Art $97(3) \ldots$. \\
\hline Art 4(1)(b) ............. & .......16, 160, 166 \\
\hline $\operatorname{Art} 4(1)(\mathrm{c}) \ldots .$. & Art $98 \ldots . . .$. \\
\hline 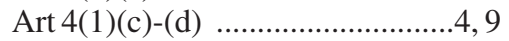 & Art $98(1) \ldots .$. \\
\hline 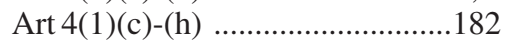 & Art $98(2)$ \\
\hline Art $4(1)(d) \ldots \ldots \ldots \ldots . .180,182-3,192-3$ & Art $100 \ldots . .$. \\
\hline Art 4(1)(e) ...................................... & Regulation 1259/2010/EU \\
\hline 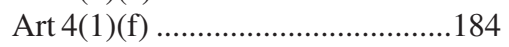 & nhanced \\
\hline Art 4(1)(g) …............6, 182, 184-5 & co-operation in the area of the law \\
\hline 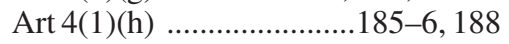 & applicable to divorce and legal \\
\hline Art 4(2) ........171-5, 177-81, 188-9, & separation (Rome III) ............. i \\
\hline $192,195-6,215-16$ & Regulation 650/2012/EU on \\
\hline Art 4(3) ....171, 173-4, 178, 180-81, & le lay \\
\hline $183-5,188-91,193,196,215$ & cement of \\
\hline Art 4(4) .......171, 174, 179, 181, 185, & decisions and acceptance and \\
\hline $193-6$ & \\
\hline Art 5 & instruments in matters of \\
\hline Arts 5-8 & succession and on the creation of a \\
\hline 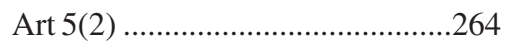 & an Certificate of \\
\hline Art $6 \ldots \ldots \ldots .6,171,180,182,184,249$ & Succession . \\
\hline $261-3,267-74,280,300$ & Regulation $1215 / 2012 / \mathrm{EU}$ on \\
\hline Art 6(1) & jurisdiction and the recognition \\
\hline Art 6(2) ..........263-4, 270, 273, 275 & and enforcement of judgments in \\
\hline Art 6(4) & civil and commercial matters \\
\hline 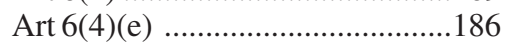 & (Brussels I revised version) ....viii, \\
\hline Art $7 \ldots \ldots \ldots . . .9-11,171,254,258,261$ & $4,6,9,15,18,23-5$, \\
\hline Art $7(1)$ & $27-9,36-7,39,68-9,75,77-8$, \\
\hline Art $7(2)$ & $80-82,86-7,91,93,96,116-17$, \\
\hline Art $7(3)$ & $123,128,144,146-7,149-50$, \\
\hline ......9, 171, 261, 277, 280, 300 & $152,155,157-9,162-3,165$, \\
\hline$\ldots .263-4,269,275$ & $167-8,268,289,296-7,322,328$, \\
\hline
\end{tabular}




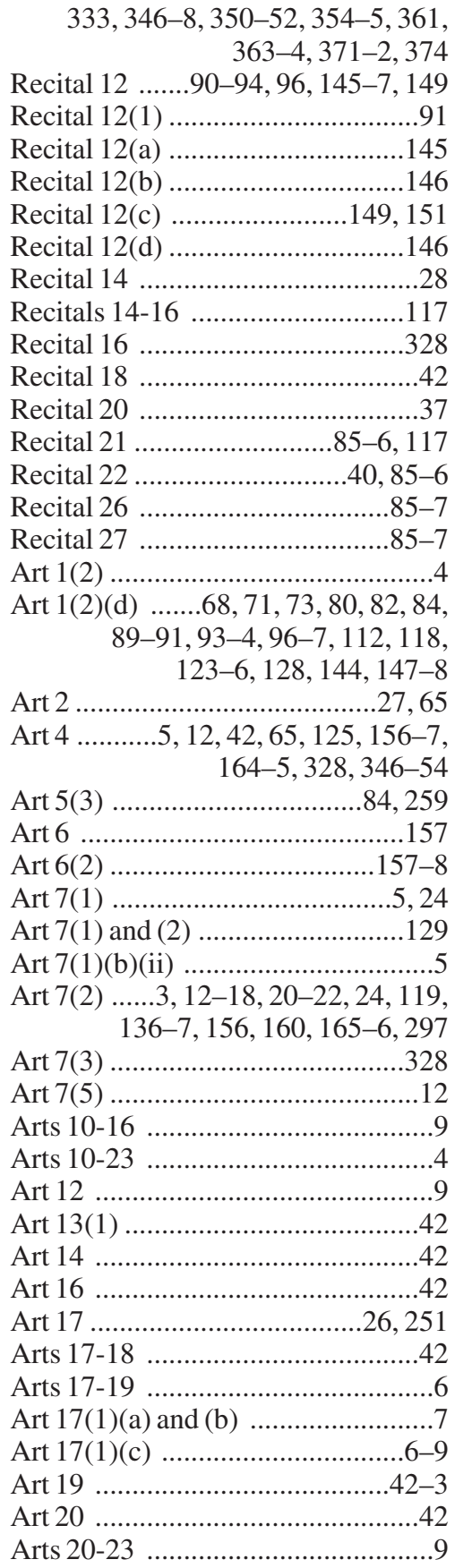

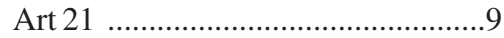

Art 24 ...................152, 161-3, 348

Art 24(1) ..................................4, 9

Art 24(2) ..................................361-4

Art 24(4) .........................................162

Art 25 ............. 5, 25-8, 32-5, 37-40,

$65-6,85,272,348$

Art 25(1) ......................................... 37

Art 25(2) ..................................34-5

Art 25(5) ...................................38-9

Art 26 ......................................5, 40

Art 26(1) ......................................85

Art 27 ....................................... 72

Arts 27-30 ...................................108

Art 28 ............................................

Art 29 ....................41, 85, 125, 167

Arts 29-34 _.............................85, 123

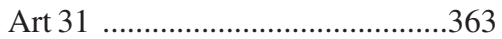

Art 31(2) ............................40-42, 85

Art 31(3) .................................38, 40

Art 32(1)(b) .................................167

Art 33(2) .......................................167

Art 33(6) .........................................167

Art 35 .................................... 125-6

Art 45(1)(a) .................................150

Art 45(1)(c) ..................................363

Art 45(1)(e) ..................................363

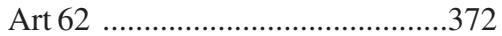

Art 63 ............................289, 372-3

Art 73(2) .....................111, 140, 151

Regulation 1257/2012/EU

implementing enhanced

co-operation in the area of the creation of unitary patent protection

Art 1(2) ............................................. 154

Art 7 ....................................... 154

Regulation 1260/2012/EU

implementing enhanced

co-operation in the area of the

creation of unitary patent

protection with regard to the

applicable translation

arrangements

Rome Convention 1980 on the Law

Applicable to Contractual

Obligations .........viiii, x, 1, 172-6,

183, 188, 190, 214, 221-2, 232-3, 


$$
\text { 235-6, 241-2, 256, 263, 272, }
$$

275-7, 282, 347

Art 1(1)

235,241

Art 4

193, 222

Art 4(1)

.172

Art 4(2)

174

Art 4(5)

$188,192-3$

Art 5

268,271

Art 7

272

Art 20

256-7

Rome I Regulation, see Regulation 593/ 2008/EC on the law applicable to contractual obligations (Rome I)

Rome II Regulation, see Regulation 864/2007/EC on the law applicable to non-contractual obligations (Rome II)

Treaty on the Functioning of the European Union

Art 18

.250

Art 19

..135

Art 49 $250,367,370$

Art 54 $.250,370$

Art 65 132

Art 81 253,313

Art 101 .264

Art 114

.253

Art 169

268

Art 267 118

\section{INTERNATIONAL}

Agreement establishing the World Trade Organisation 1994 ........306

Ann 1A .198

Agreement on a European Economic

Area .265

Agreement on Trade-Related Aspects of Intellectual Property Rights 1994. $168-9$

Art 41 169

Convention on International Sale of

Goods (CISG) 1980 ..xi, 199-202, 204-11, 213, 216-17, 219, 225, 233-4, 236-7, 247, 264

Art 1 $202,231,234$

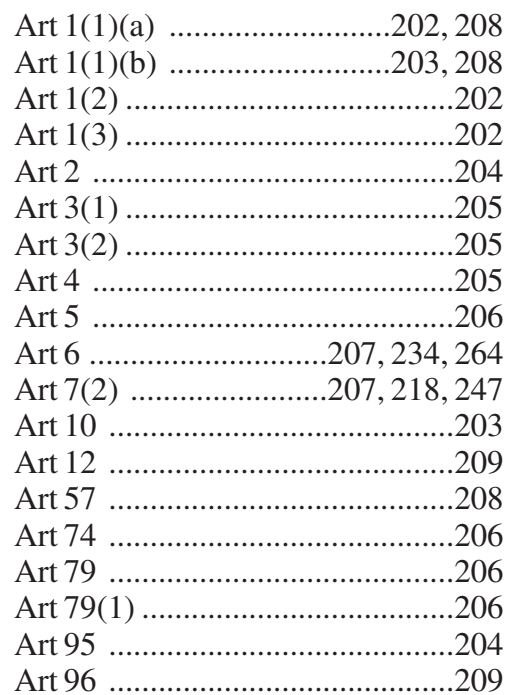

European Convention on Human

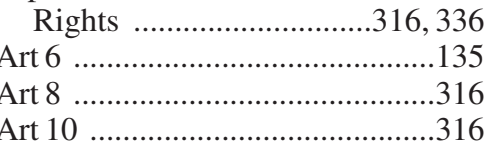

European Convention on International Commercial Arbitration 1961, Art VII .....................................239

European Patent Convention, see Munich Convention on the Grant of European Patents 1973

General Agreement on Tariffs and Trade (GATT) 1947 197

Hague Convention 1971 on the Law Applicable to Traffic

Accidents .287

Hague Convention 1996 on

Jurisdiction, Applicable Law,

Recognition, Enforcement and

Co-operation in respect of Parental

Responsibility and Measures for

the Protection of Children ..........ix

Hague Convention 2007 on the International Recovery of Child Support and Other Forms of Family Maintenance ix

Hague Protocol 2007 on the Law Applicable to Maintenance Obligations ix $-\mathrm{X}$ 
Hamburg Rules, Art 2(1)(e) .231

International Convention for the Unification of Certain Rules of Law Relating to Bills of Lading, 1968 214,231

Art X(c)

Lugano Convention 1988 on Jurisdiction and the Enforcement of Judgments in Civil and Commercial Matters ..viii

Lugano Convention 2007 on Jurisdiction and the Recognition and Enforcement of Judgments in Civil and Commercial Matters viii, $x, 374$

Munich Convention on the Grant of European Patents 1973 .153 Art 6(1) .154

Art 60 $152-4$

New York Convention, see United Nations Convention on the Recognition and Enforcement of Foreign Arbitral Awards, New York, 10 June 1958

Principles of European Contract Law (PECL) $218,221,226,228$, 243, 264

UNIDROIT Principles of International Commercial Contracts ......xi, 200, 210, 217-21, 226, 228, 233, 243,264

Uniform Customs and Practice for Documentary Credits (UCP) 600 .........226-30, 237, 243

Art 1 eUCP, Art e2(a) .228

Uniform Law on Formation of International Sales 1964 (ULFIS) 200, 209-11

Uniform Laws on International Sales 1964 (ULIS) 200, 209-11

United Nations Convention on

Contracts for the International

Carriage of Goods Wholly or

Partly by Sea 2008

United Nations Convention on the

Recognition and Enforcement of

Foreign Arbitral Awards, New
York, 10 June 1958 69 89-91, 93, 97-9, 103-7, $111,119,121,123,126$, 139-40, 149-51

Art I .97-8, 107

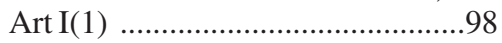

Art II 128

Art II(1) . .91

Art II(3) ....91, 106, 121, 131, 139-40

Art III . .99

Art V 97-9, 101

Art V(1) . .99

Art V(1)(a) . .99

Art V(1)(b) .99

Art V(1)(e) ..93, 99-100, 108-9

Art V(1)(f) . .99

Art V(2) . .99

$\operatorname{Art~V(2)(a)~}$ 150

Art V(2)(b)

Art V(a)(1) 150

Art VII $.93,97,99,111$

\section{NATIONAL}

\section{Algeria}

Civil Code 210

\section{Belgium}

Code of Private International Law Art 93 152 Art 94 152

Judicial Code

Art 1697(1) $102-3$

Sixth Part: Arbitration (adopted 4 July 1972 and last amended on 19 May 1998)

\section{Canada}

Civil Code of Quebec 210 Art 3126 295 
xxviii Research handbook on EU private international law

\section{China}

Foreign Economic Contract Law, 1985

\section{France}

Arbitration Act, Art 1448

Civil Code 142 Art 14 157,235

Civil Procedure Code, Art 1502(1) ..101

Decree No. 2011-48 of 13 January 2011, Ch III

\section{Germany}

EGBGB, Art 40(2)

\section{Greece}

Law on Copyright, Art 67

\section{Hong Kong}

Arbitration Ordinance of Hong Kong,

$$
\text { s. } 34
$$

\section{Italy}

Arbitration Act, Art 819

\section{Netherlands}

Civil Code

\section{Russia}

Federation Constitution, Art 15(4) ..204

\section{Switzerland}

Private International Law Act 1987

Art 133

Art 186

Art 192

\section{United Kingdom}

Arbitration Act 1996 ................114, 118

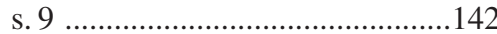

s. 18(2) ..........................................125

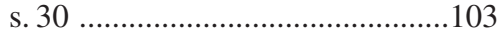

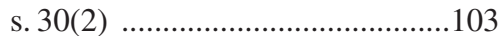

s. 46 ...................................2223, 239

s. 66 .............................................. 119

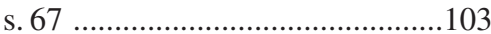

s. 69 ..............................119-20, 148

Carriage of Goods by Sea Act 1971,

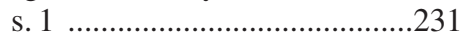

Consumer Protection Act 1987 .........310

Defamation Act 2013 .......316, 328, 333

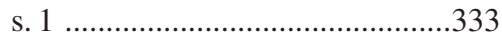

s. 1(2) ………………...................333

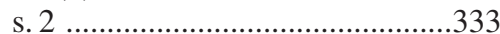

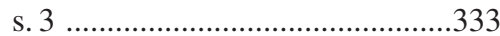

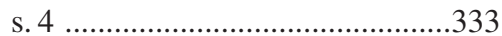

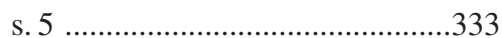

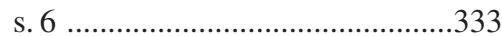

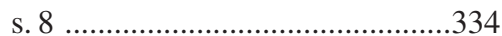

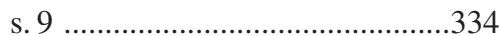

s. 9(2) ……....................................334

s. 11 .............................................333

Private International Law

(Miscellaneous Provisions) Act

1995 .......................287, 311, 323

Pt III ..................................287, 309

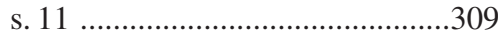

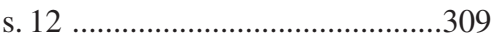

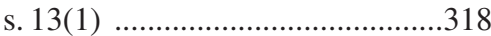

Sale of Goods Act 1979 ...................208

s. 2(1) ..........................................201

Scotland

Arbitration Rules, r.19 .................103

Senior Courts Act 1981, s. 37 ..........114

Supreme Court Act 1981 ....................76

Uniform Law on International Sales Act 1967, s. 1(3) .209

\section{United States}

\section{US Code}

§ 12(b)(3) .....................................62

$\S 1332$ 
Table of legislation and analogous instruments $\mathrm{xxix}$

\begin{tabular}{|c|c|}
\hline$\S 1404(a) \ldots \ldots \ldots \ldots \ldots \ldots \ldots \ldots . . .55,60-62$ & $\begin{array}{r}\text { ouisiana Civ } \\
\text { Art } 3544\end{array}$ \\
\hline$\S 1406(a)$ & Art 3544(1) .. \\
\hline lien Tort Clain & Restaten \\
\hline $\begin{array}{l}\text { Civil Code, } \\
\text { g. .................... }\end{array}$ & \\
\hline arria &. .30 \\
\hline .46 & \\
\hline s 3(8) & ercial Code \\
\hline
\end{tabular}


Peter Stone and Youseph Farah - 9781781954553 Downloaded from PubFactory at 04/26/2023 09:19:18AM via free access 